\title{
Neuroplasticity and amblyopia: vision at the balance point
}

Vijay K. Tailor ${ }^{1}$

D. Samuel Schwarzkopf ${ }^{2}$

Annegret H. Dahlmann-Noor ${ }^{1}$

${ }^{1}$ Department of Paediatric Ophthalmology and Strabismus, NIHR Biomedical Research Centre at Moorfields Eye Hospital and UCL Institute of Ophthalmology, 162 City Road, London EC1V 2PD, UK

2 UCL Experimental Psychology, 26 Bedford Way, London WC1H 0AP \& UCL Institute of Cognitive Neuroscience, 17 Queen Square, London WC1N 3AZ

Corresponding author:

A H Dahlmann-Noor Department of Paediatric Ophthalmology and Strabismus

NIHR Biomedical Research Centre at Moorfields Eye Hospital and UCL Institute of Ophthalmology 162 City Road

London EC1V 2PD UK

Phone 00442075662013 e-mail annegret.dahlmann-noor@moorfields.nhs.uk

This work was not supported by dedicated funding. 
Abstract Purpose: New insights into triggers and brakes of plasticity in the visual system are being translated into new treatment approaches which may improve outcomes not only in children, but also in adults.

Recent findings: Visual experience-driven plasticity is greatest in early childhood, triggered by maturation of inhibitory interneurons which facilitate strengthening of synchronous synaptic connections, and inactivation of others. Normal binocular development leads to progressive refinement of monocular visual acuity, stereoacuity and fusion of images from both eyes. At the end of the "critical period", structural and functional brakes such as dampening of acetylcholine receptor signalling and formation of perineuronal nets limit further synaptic remodelling. Imbalanced visual input from the two eyes can lead to imbalanced neural processing and permanent visual deficits, the commonest of which is amblyopia.

Summary: The efficacy of new behavioural, physical and pharmacological interventions aiming to balance visual input and visual processing have been described in humans, and some are currently under evaluation in randomised controlled trials. Outcomes may change amblyopia treatment for children and adults, but the safety of new approaches will need careful monitoring, as permanent adverse events may occur when plasticity is re-induced after the end of the critical period.

\section{Supplementary Digital Content: video abstract}

\section{Keywords}

Neuronal plasticity

Amblyopia

Visual perception

Amblyopia, Suppression

\section{Glossary}

anisometropia two

a difference in refractive error (spectacle prescription) between the eyes a-tDCS anodal transcranial direct current 
stimulation $\mathrm{Cl}$

Balance point

signal

Bangerter filter

image

Binocular viewing

cTBS

Dichoptic viewing confidence interval

the point where signals from the two eyes have equal weighting, and integration is normal

filter composed of microelements which distort the perceived

Viewing with both eyes simultaneously

continued theta burst stimulation

Viewing of different images shown to right and left eye fMRI

functional magnetic resonance imaging

GABA gamma-Aminobutyric acid

IOCSR

inter-ocular contrast sensitivity ratio, a measure of the imbalance in contrast sensitivity between an amblyopic and better-seeing eye logarithm of the minimum angle of resolution; contemporary

$\log$ MAR visual acuity or spatial resolution luminance

measure of

here the perceived light intensity; reduced by NDF, red filters, Bangerter

filters, which all reduce the amount of light that passes through the

filter Monocular viewing Viewing with one eye only

NDF

simulate

amblyopia;

by the better-

NMDA

Perceptual learning

possible

approach

practice of visual

population Receptive Field

RCT

rTMS

Sbisa bar

the

point SD

error Suppression

eye randomized controlled trial

repetitive transcranial magnetic brain stimulation

Neutral density filter; placed in front of the better-seeing eye to amblyopia or to determine the balance point in individuals with also proposed for therapeutic use to degrade the image seen seeing eye to balance visual input

$\mathrm{N}$-methyl-D-aspartate

Improving performance of a trained task by repeated practice, with transfer of functional gain to other functions; here a treatment involving occlusion of the better-seeing eye and repeated tasks using the amblyopic eye pRF

Red filter bar; filters increasingly reduce luminance; if placed in front of better-seeing eye, simple method to approximate the balance Standard deviation SE Standard inhibitory influence of the better-seeing eye over the amblyopic when both eyes are viewing 


\section{Main Text}

Introduction Over recent years, insights into visual development and deficits in visual processing in amblyopia have led to new treatment approaches. This review aims to provide a concise update on advances in the understanding of plasticity in the visual system, and new treatment approaches which harness plasticity to improve visual function in amblyopia. Were possible, we refer to work published in 2015-16; to set new findings into the appropriate context we refer to primary research and comprehensive review articles.

Plasticity in the visual system is greatest in early infancy. The architecture of the visual cortex, including the arrangement of neurons from each eye in vertical "ocular dominance" columns, and retinotopic horizontal organisation with neighbouring cells processing signals from receptive fields close to each other in the visual fields, is present at birth, i.e. develops under the guidance of genetic programs and without visual experience [1]. After birth, the pre-shaped architecture is refined by visual experience, with strengthening of synaptic connections between neurons that fire at the same time, and pruning of synapses that are not synchronised [2]. With progressive outgrowth and maturation of axons and dendrites, horizontal connections develop between neurons, and neural networks form, reflecting functional circuits or "maps" [3]. Plasticity, the ability of neurons to adapt and change in response to stimuli, is highest in young children, a period termed "critical period of vision development" (Fig. 1).

This phase is triggered by maturation of inhibitory, GABA-producing interneurons such as parvalbumin-positive large basket cells, which extend axons around excitatory neurons, modulating their signalling and setting up an excitatory-inhibitory "balance" [2, 4-6].

Structural and functional "brakes" where for long considered to mediate the end of the critical period, by limiting further synaptic remodelling. Examples are perineural nets, primarily composed of chondroitin sulfate proteoglycans, molecules which modulate the response to neuro-transmitters at synapses, such as Lynx1, a molecule which dampens the effect of acetylcholine, and Nogo-66-receptor-mediated myelin inhibition of neurite outgrowth $[2,7,8]$. However, a recent study showed that experience-dependent maturation of silent excitatory synapses onto pyramidal neurons in the primary visual cortex (V1) terminates the critical period of plasticity, and that this pathway is independent of the inhibitory tone of GABAergic interneurons [6]. Maturation of processing in V1 and extrastriate areas is accompanied by refinement of efferent pathways, leading to increasing stability of central fixation and ocular alignment.

Imbalanced visual input Disruption of visual input during the critical period, for example by temporary occlusion of one eye, results in permanent visual deficits $[9,10]$. In humans, disturbance of visual input in early childhood causes amblyopia, which affects $2-4 \%$ of individuals. Amblyopia is usually associated with a difference in refractive error (spectacle prescription) between the two eyes (anisometropia), misalignment of the eyes (strabismus), or both. Rarely, opacities of the cornea, crystalline lens (congenital/infantile cataract) or vitreous can cause amblyopia by depriving the retina of a clear image. Amblyopia is usually characterised by reduced vision in one eye, though bilateral cataract or hypermetropia (longsightedness) can cause bilateral amblyopia. Amblyopia not only affects acuity, but also contrast sensitivity and fixation stability of the affected eye, and causes reduced stereoacuity (3D vision) and increased vulnerability to "crowding", difficulty identifying a shape surrounded by visual "clutter" [11,12]. Imbalanced visual processing However, it is not only the processing of signals from the amblyopic eye that is impaired, there are also deficits in binocular processing, such as "suppression" [13, 14], an inhibitory influence of the better-seeing eye over the amblyopic eye when both eyes are viewing [15]. Suppression is considered an adaptive response to imbalanced visual input, preventing double vision in strabismus and visual confusion in anisometropia. The mechanisms underlying suppression are not fully known; models imply inhibitory circuits and neurotransmitters [16]. Various measures of suppression have been developed. Vision 
scientists commonly measure the inter-ocular contrast sensitivity ratio (IOCSR), which reflects decreased contrast sensitivity in the amblyopic eye and typically correlates with amblyopic eye acuity [17]. The terms suppression and "abnormal IOCSR" are often used interchangeably [18]. However, whilst under normal viewing conditions, suppression appears to explain amblyopic vision deficits, binocular summation is normal when the contrast of images shown to the better-seeing eye matches the contrast sensitivity of the amblyopic eye [19]. This is the "balance point", the point where signals from both eyes have equal weighting [15, 20,21]. Data from adults with true and simulated amblyopia (placement of neutral density filters, NDF, in front of one eye) have been used to develop computational models of neural processing [21-24]. One such model indicates that excitatory signals from the amblyopic eye are attenuated and abnormal noise is increased, whilst binocular summation and inhibition are intact $[21,22,25]$. Suppression may correspond to the "attenuator" in this model, and may equate to a long-term sensitivity reduction, rather than a transient, active process of inhibition [22]. The observed attenuation may reflect reduced synchronisation of neural firing [22].

\section{Cutting edge neuro-imaging demonstrates changes in cortical structure and functional organization in amblyopia. In addition to animal models and human psychophysical studies, functional magnetic resonance imaging (fMRI) has delivered new insights into the biological factors underlying amblyopia and neural plasticity in humans. fMRI studies of amblyopia are challenging, as careful matching of visual acuity and control over eye movements are required; the latter is hampered by amblyopic eye fixation instability. Population receptive field (pRF) mapping estimates the receptive field size of cortical neurons, i. e. the size of the region in visual space in which a stimulus will trigger the firing of that neuron. The pRF sizes of neurons processing signals from the amblyopic eye in adults with strabismic amblyopia are consistently enlarged [26] (Fig 2) .}

\section{Figure 2. Non-invasive functional MRI demonstrates structural and functional changes in}

amblyopia. Top left: Population receptor field $(p R F)$ mapping estimates the receptive field size of cortical neurons, i. e. the size of the region in visual space in which a stimulus will trigger the firing of that neuron. The colours indicate the different pRF sizes as shown in the color bar. A, B: medial and lateral view of pRF size map for one individual. Solid lines demarcate visual field maps V1 and V3 (from [27]). Top right: In children with anisometropic amblyopia, diffusion tensor imaging shows thickening of the calcarine cortex (blue), which may indicate reduced "pruning" of neurons (from [28]). Bottom: In the same children, there is reduced integrity of the optical radiations (right) compared with healthy controls (left) (from [28]).

Importantly, this change not only affects $\mathrm{V} 1$ but also extrastriate regions. It may arise from reduced spatial resolution or greater spatial scatter of neuronal receptive fields [26].

Diffusion tensor imaging shows that in children with amblyopia, the integrity of the optical radiations is reduced, as is the cortical thickness of several higher visual areas (Fig 2)[28]. Interestingly, the calcarine cortex (which contains V1) appears thicker in individuals with amblyopia, which could indicate insufficient pruning, as is also seen in congenitally blind individuals [29, 30]. Differences in white matter integrity in amblyopia are also not restricted to the optic radiations but are also found in cortico-cortical tracts, including the corpus callosum and links between visual and temporal and frontal cortex [31, 32]. 


\section{Current amblyopia treatment forces use of the amblyopic eye and works best in}

young children. Treatment aims to remove the causes of imbalanced visual input, by correcting any refractive error by glasses, and by surgically aligning the eyes to potentially improve binocular processing. Part-time occlusion or pharmacological blurring of near vision of the better-seeing eye with atropine eye drops promote processing of signals from the amblyopic eye [33]. Treatment is most successful before the age of 7 to 8 years: in younger children with moderate amblyopia an improvement in amblyopic eye acuity of 0.25 to 0.30 logMAR (two and a half to three lines on an acuity chart) can be expected after four months of treatment [34,35]; in those with severe amblyopia, improvement is around $0.50 \log M A R$ [36]. In older children and adults, the effect is less, for both moderate and severe amblyopia: 0.15 to 0.18 logMAR [37, 38]. For long this decline in response to treatment has been taken to indicate that the "sensitive period for treatment" is identical to the "critical period of vision development". However, animal models have now successfully induced juvenile-like plasticity and restored vision in adult animals, and new amblyopia treatments have shown vision improvement in some adults (see below). Strategies to balance visual input and/or enhance neural plasticity to increase processing of signals from the amblyopic eye and binocular integration may improve treatment outcomes after the sensitive period. New treatments for amblyopia balance visual input and transiently increase plasticity. A range of behavioural, physical and pharmacological methods have been developed. Some have been evaluated in animal models only, others have been tested in human studies; few have moved to randomised controlled trials (RCT).

\section{Behavioural approaches}

a. a) Balancing visual input: computer-based dichoptic image presentation The observation that in amblyopia, binocular integration at the "balance point" is normal, led to the development of an "anti-suppression" treatment [39], using a "falling block" computer game (Tetris $\left.{ }^{\top \mathrm{M}}\right)$. Images are presented dichoptically, with contrast at the balance point, strengthening binocular summation: blocks are presented at high contrast to the amblyopic eye and low contrast to the better-seeing eye. Different parts of the visual scene are shown to each eye, and successful game play requires use of the information shown to the amblyopic eye only. Contrast detection thresholds in the amblyopic eye gradually improve [39], and the contrast of the image shown to the better-seeing eye can be increased. In some cases, normalisation of visual processing, including acuity and stereoacuity, have been reported. Activity-dependent changes in neural activity and threshold modification enabling amblyopic eye signals to induce long-term potentiation, the basis of synaptic strengthening and plasticity, may mediate these effects [15]. The hardware for dichoptic viewing has evolved to accommodate different patient groups - small devices such as iPods for adults [40], larger iPads for children [41, 42]. Case series show promising results with acuity improvements of around $0.24(95 \% \mathrm{Cl}=0.04) \log$ MAR [43-45]. Two RCTs have recently been completed (NCT02200211 and ACTRN12613001004752); publication of results is pending. The efficacy of this approach may be enhanced by simultaneous anodal transcranial direct current stimulation (a-tDCS, see below), with greater stereoacuity improvement than dichoptic treatment alone and with mean acuity improvement of $0.34 \log M A R[46]$.

A similar approach shows a blurred image to the better-seeing eye, the level of blur reducing acuity in the fellow eye to that of the amblyopic eye ("binocular balanced viewing"). In 22 children age 3-11 years watching blur-matched movies for an hour a day achieved a mean acuity improvement of 0.27 (SD 0.22) logMAR ([47] Bossi, unpublished data). Interestingly, changes in visual acuity did not systematically correlate with changes in IOCSR, lending support to the notion that binocular suppression mechanisms are not the only factors involved in amblyopia.

Recently, a dichoptic video action game matching luminance and contrast has been combined with a monocular form of amblyopia treatment, "perceptual learning" 
(PL) (see below). The same image was shown to both eyes, but the image to the better-seeing eye had reduced luminance/contrast. Acuity improved by a mean of 0.14 (SD 0.01) logMAR in adults and was sustained for two months, interestingly without systematic correlation between visual acuity improvement and IOCSR [48].

a. a) Balancing visual input: optical methods to degrade input from betterseeing eye

Bangerter or NDF can be applied to glasses to reduce input from the better-seeing eye. Bangerter filters are made of microelements which distort the perceived image and can reduce IOCSR and normalise binocular contrast summation [49]. NDFs reduce the perceived luminance, and in individuals with amblyopia can improve binocular combination [50]. NDFs can be graded to match the visual acuity of the amblyopic eye [51]. Both NDF and Bangerter filters work by delaying visual signaling; it is unclear whether this merely simulates the degraded signaling from an amblyopic eye, or whether it may induce additional temporal dissociation and reduce binocular co-operation further [15, 17].

a. b) Non-balancing dichoptic methods Other methods use dichoptic presentation of images with the same level of contrast and clarity. One example is the Interactive Binocular Treatment (iBiT) approach, which presents some parts of a visual scene to the better-seeing eye, and other parts, critical for successful game play or video watching, to the amblyopic eye. Initial case series showed acuity improvement of 0.18 (SD 0.14) logMAR [52]; however, a recent RCT indicated lesser efficacy (0.06 to 0.10 logMAR) [53].

b. c) Monocular methods Perceptual learning In the original form of PL, the better-seeing eye is occluded whilst the amblyopic eye is viewing increasingly challenging stimuli [54], to improve performance of a trained task by repeated practice, involving multiple, in some examples hundreds, of sessions, with tens of thousands of "trials" or learning episodes [55]. This can improve contrast sensitivity in adults with amblyopia [56]; gain transfers to acuity (improvements of around 0.24 logMAR) [57] and binocular function [54, 58]. The underlying mechanism may include reduction of internal noise or more efficient use of stimulus information in the primary visual cortex and higher areas involved in attention and decision making ("retuning the perceptual template") $[54,55,59]$. Improvements last for up to 12 months after discontinuation of treatment [55]. The PL approach is currently being evaluated in RCTs (NCT01223716, NCT01115283). Off-the shelf video action games Video action games, which ask the player to perform repeated tasks to progress in a game, have been explored as a different means to induce visual learning, though the stimuli presented by off-the-shelf games are not specific and controlled. Indeed, playing an off-the shelf video game for two hours a day using only the amblyopic eye can improve acuity by around $0.16 \log M A R$, as well as stereopsis and other aspects of visual function [60]. The mechanism may include an enhanced ability to learn ("enhanced perceptual templates"), transferrable to visual tasks [61].

c. d) Other methods Two studies reported surprising and at first sight counterintuitive results. Short-term (2.5 hours) patching of the amblyopic eye induces a transient (30 minute) increase in amblyopic eye signalling when the patch is removed. This may reflect homeostatic intrinsic plasticity which regulates an increase of contrast gain in the patched eye, a reduction in suppression, and/or a reduction in neural noise [62].

Secondly, a recent study showed that exposure to complete darkness can "re-set" neural signalling and normalise vision in previously amblyopic eyes in kittens [63]. Darkness exposure re-induces high levels of neuroplasticity, reflected in significant reduction of neurofilament light subunit expression in visual cortex neurons [63].

\section{Physical methods}

Repetitive transcranial magnetic brain stimulation (rTMS), continued theta burst stimulation (cTBS) and anodal transcranial direct current stimulation (a-tDCS) 
transiently alter neuronal activity. Targeting imbalanced cortical processing, exerting an excitatory effect on inhibited amblyopic eye signalling, rTMS induced an improvement of contrast detection thresholds by $40 \%$ for about a week in adults with amblyopia [64]. Regular daily application of theta bursts (continued theta burst stimulation = cTBS) over five days had a cumulative effect which lasted for over two months [65]. cTBS is delivered in short sessions of 40 seconds, compared with 10 to 15 minutes for rTMS. Similarly, a-tDCS transiently improves contrast sensitivity and stereopsis and normalises visual cortex activation for 30 minutes in some adults with amblyopia [66]. These physical methods presumably increase excitability of projections from the amblyopic eye, with reduction in GABA and increase in glutamate concentration, or may reduce noise in amblyopic eye signalling $[65,66]$.

\section{Pharmacological and cellular methods}

Four pharmacological agents have been shown to enhance visual cortex plasticity in animal models, and three of these have been or are currently being investigated in RCTs. An open-label RCT reported that Citicoline enhances the effect of occlusion treatment in children age 4-13 years [67], with mean acuity improvement of $0.20 \log M A R$ for up to a year. Citicoline increases levels of dopamine and acetylcholine in the central nervous system. Levodopa (L-dopa), combined with carbidopa to block peripheral and enhance central Ldopa conversion to dopamine, transiently improves visual function in individuals with amblyopia, but a recent RCT with older children concluded that there was there was no additional benefit over occlusion treatment only [68].

As reversal of Lynx1-mediated dampening of acetylcholine receptor signalling re-induces

plasticity in mice [8], the effect of donepezil, a centrally acting acetylcholinesterase inhibitor, on human amblyopia is currently the topic of a RCT (NCT01584076). Fluoxetine, a selective serotonine reuptake inhibitor commonly used in depression, re-induces visual plasticity in rats and can reverse monocular deprivation-induced visual loss [69], possibly by creating a novel synaptic environment that favours mature NMDA and GABA-A receptor subunits [70]. However, this concept has not progressed to human studies.

Figure 3 and Table 1 provide an overview of novel treatment approaches for human amblyopia.

Other approaches, explored in animal studies only, are the transplantation of inhibitory interneuron precursors (in young mice after the end of the critical period, this triggers a second critical period [71]; in adult animals, it re-activates a juvenile-type of plasticity [72]), and blockade of paired immunoglobulin-like receptor B (PirB) by infusion of a soluble PirB ectodomain into mouse visual cortex (triggering formation of new functional synapses [73]). Lastly, cortical injections of chondroitinase dissolve extracellular matrix, specifically chondroitin sulfate proteoglycans which surround neurons and inhibit cell migration and axon growth from the end of the sensitive period. This restores vision in adult rats exposed to monocular deprivation [74], but in cats only induces structural changes, but no significant functional recovery [75]. Treating amblyopia after the end of the sensitive period may trigger permanent adverse effects.

There are concerns that treatment of amblyopia after the sensitive period may cause unintended adverse effects, particularly double vision (diplopia) in individuals with strabismic amblyopia. In individuals who have not developed sensory fusion mechanisms to combine corresponding images from the two eyes, treatments which reduce suppression can cause double vision, which in extreme cases may be "intractable", i.e. cannot be alleviated by surgery or prisms [76, 77]. Incidence or risk of this adverse event are not known. Some 
recommend monitoring of suppression with NDF or red filter (Sbisa) bars [78, 79], although the link between suppression and acuity is unclear [48, 80] (Bossi, unpublished data). Even less is known about the risks to neuronal health from pharmacological or cellular re-induction of cortical plasticity [2]. Conclusion Pre-clinical and clinical studies demonstrate that balancing binocular visual input may open new treatment options for adults and children with amblyopia. Physical and pharmacological approaches may enhance treatment efficacy by further increasing synaptic strength and remodeling. Further work is required to improve outcomes and to monitor the safety of new approaches. 


\section{Key points}

- $\quad$ Psychophysiological and neuro-imaging studies have begun to clarify

functional and structural changes in visual processing in amblyopia.

- $\quad \square \quad$ New behavioural treatments balancing visual input may improve outcomes of amblyopia treatment in children and adults.

- $\quad \square \quad$ Physical approaches can transiently increase plasticity in the adult visual cortex, and may enhance the efficacy of behavioural treatments.

- $\quad \square \quad$ Pharmacological approaches to transiently increase visual cortex plasticity may equally enhance the efficacy of behavioural treatments.

- $\quad \square \quad$ The safety of new approaches needs to be monitored, both in terms of shortterm visual adverse events such as permanent double vision, and, for pharmacological and cellular plasticity-enhancing methods, neuronal health.

\section{Acknowledgements}

Figure 1 and 3 were drawn using the Motifolio collection of biomedical diagrams. We thank Prof. S. Dakin for contributing an illustration of the binocular balanced viewing approach for Figure 3. Financial support and sponsorship This review was not supported by specific funding. AHDN and VT are employed by the NIHR Biomedical Research Centre for Ophthalmology at Moorfields Eye Hospital and UCL Institute of Ophthalmology. AHDN's current work is funded by grants from Moorfields Eye Charity, the British Council for the Prevention of Blindness, Action for Medical Research and the Wates Foundation.

The views expressed in this publication are those of the authors and not necessarily those of the Department of Health. Conflicts of interest AHDN and VT contribute to the clinical evaluation of Balanced Binocular Viewing; AHDN is lead applicant on a pending grant application for a pilot RCT of this technology.

\section{References}

Figure titles and legends Figure 1. Plasticity (blue line) is highest during the first months of life, when visual experience shapes neural circuits. Inhibitory interneurons such as parvalbumin-positive basket cells make connections with excitatory visual cortex neurons, modulating their synaptic activity and triggering the start of the "critical period of visual plasticity". Synaptic connections between neurons who signal in tandem are strengthened, and synaptic connections that are not synchronised are "pruned". With increasing cortical maturation, functional and structural "brakes" reduce plasticity, for example Lynx-1 binding to acetylcholine receptors, deposition of extracellular matrix molecules such as chondroitin sulfate proteoglycans, which form perineuronal nets, and Nogo-66-receptor-mediated myelin inhibition of neurite outgrowth. Experience-dependent maturation of initially silent, excitatory synapses terminates the critical period of plasticity.

\section{Figure 2. Non-invasive functional MRI demonstrates structural and functional changes in}

amblyopia. Top left: Population receptor field (pRF) mapping estimates the receptive field size of cortical neurons, i. e. the size of the region in visual space in which a stimulus will trigger the firing of that neuron. The colours indicate the different pRF sizes as shown in the color bar. A, B: medial and lateral view of pRF size map for one individual. Solid lines demarcate visual field maps V1 and 
V3 (from [27]). Top right: In children with anisometropic amblyopia, diffusion tensor imaging shows thickening of the calcarine cortex (blue), which may indicate reduced "pruning" of neurons (from [28]). Bottom: In the same children, there is reduced integrity of the optical radiations (right) compared with healthy controls (left) (from [28]).

Figure 3. New treatment approaches for amblyopia balance visual input and/or increase visual cortex plasticity. Top left: matching the contrast or blur of the image shown to the better seeing eye to the perception of the amblyopic eye brings visual input to the "balance point".

Strategies include contrast-matched Tetris games (anti-suppression treatment, left) and watching blur-matched movies (balanced binocular viewing, right). Top right: transcranial currents and drugs can temporarily induce neuronal plasticity and allow preferential processing of signals from the amblyopic eye. Bottom left: repeated practice of highly specific visual tasks (perceptual learning) or repetitive game play using the amblyopic eye only can improve visual function. Bottom right: game play or movie watching requiring combination of images from right and left eye may improve vision in the amblyopic eye. The figure illustrating the balanced binocular viewing approach (top left) contains an image from the movie "Big Buck Bunny", used under a Creative Commons Attribution 3.0 license, and kindly contributed by S. Dakin.

Table 1. Overview of new treatment approaches for amblyopia and visual outcomes.1. Mitchell DE, Duffy KR. The case from animal studies for balanced binocular treatment strategies for human amblyopia. Ophthalmic Physiol Opt. 2014;34(2):12945.

2. Hensch TK. The Power of the Infant Brain. Sci Am. 2016;314(2):64-9. 3. White LE, Fitzpatrick D. Vision and cortical map development. Neuron. 2007;56(2):327-38.

4. Hensch TK, Fagiolini M, Mataga N, Stryker MP, Baekkeskov S, Kash SF. Local GABA circuit control of experience-dependent plasticity in developing visual cortex. Science (New York, NY. 1998;282(5393):1504-8.

5. Nahmani M, Turrigiano GG. Adult cortical plasticity following injury: Recapitulation of critical period mechanisms? Neuroscience. 2014;283:4-16.

6. Huang X, Stodieck SK, Goetze B, Cui L, Wong MH, Wenzel C, et al. Progressive maturation of silent synapses governs the duration of a critical period. Proceedings of the National Academy of Sciences of the United States of America. 2015;112(24):E3131-40.

7. McGee AW, Yang Y, Fischer QS, Daw NW, Strittmatter SM. Experience-driven plasticity of visual cortex limited by myelin and Nogo receptor. Science (New York, NY. 2005;309(5744):2222-6.

8. Morishita H, Miwa JM, Heintz N, Hensch TK. Lynx1, a cholinergic brake, limits plasticity in adult visual cortex. Science (New York, NY. 2010;330(6008):1238-40. 
9. Hubel DH, Wiesel TN. Effects of Monocular Deprivation in Kittens. Naunyn Schmiedebergs Arch Exp Pathol Pharmakol. 1964;248:492-7.

10. Hubel DH, Wiesel TN. The period of susceptibility to the physiological effects of unilateral eye closure in kittens. The Journal of physiology. 1970;206(2):419-36.

11. Anderson EJ, Dakin SC, Schwarzkopf DS, Rees G, Greenwood JA. The neural correlates of crowding-induced changes in appearance. Curr Biol. 2012;22(13):1199206.

12. Greenwood JA, Tailor VK, Sloper JJ, Simmers AJ, Bex PJ, Dakin SC. Visual acuity, crowding, and stereo-vision are linked in children with and without amblyopia. Investigative ophthalmology \& visual science. 2012;53(12):7655-65.

13. von Graefe A. Das Sehen der Schielenden. Eine ophthalmologischphysiologische Studie.1896.

14. Worth C. Squint: its causes, pathology and treatment. 3rd ed. co Bs, editor. Philadelphia1906.

15. Hess RF, Thompson B, Baker DH. Binocular vision in amblyopia: structure, suppression and plasticity. Ophthalmic Physiol Opt. 2014;34(2):146-62.

16. Sengpiel F, Jirmann KU, Vorobyov V, Eysel UT. Strabismic suppression is mediated by inhibitory interactions in the primary visual cortex. Cerebral cortex. 2006;16(12):1750-8.

17. Li J, Hess RF, Chan LY, Deng D, Yang X, Chen X, et al. Quantitative measurement of interocular suppression in anisometropic amblyopia: a case-control study. Ophthalmology. 2013;120(8):1672-80.

18. Li J, Thompson B, Lam CS, Deng D, Chan LY, Maehara G, et al. The role of suppression in amblyopia. Investigative ophthalmology \& visual science. 2011;52(7):4169-76.

19. Baker DH, Meese TS, Mansouri B, Hess RF. Binocular summation of contrast remains intact in strabismic amblyopia. Investigative ophthalmology \& visual science. 2007;48(11):5332-8.

20. Mansouri B, Thompson B, Hess RF. Measurement of suprathreshold binocular interactions in amblyopia. Vision research. 2008;48(28):2775-84.

21. Baker DH, Meese TS, Hess RF. Contrast masking in strabismic amblyopia: attenuation, noise, interocular suppression and binocular summation. Vision research. 2008;48(15):1625-40.

22. Baker DH, Simard M, Saint-Amour D, Hess RF. Steady-state contrast response functions provide a sensitive and objective index of amblyopic deficits. Investigative ophthalmology \& visual science. 2015;56(2):1208-16.

23. Ding J, Sperling G. A gain-control theory of binocular combination.

Proceedings of the National Academy of Sciences of the United States of America. 2006;103(4):1141-6.

24. Ding J, Levi DM. Binocular contrast discrimination needs monocular multiplicative noise. Journal of vision. 2016;16(5):12.

25. Meese TS, Georgeson MA, Baker DH. Binocular contrast vision at and above threshold. Journal of vision. 2006;6(11):1224-43.

26. Clavagnier S, Dumoulin SO, Hess RF. Is the Cortical Deficit in Amblyopia Due to Reduced Cortical Magnification, Loss of Neural Resolution, or Neural Disorganization? J Neurosci. 2015;35(44):14740-55.

27. Dumoulin SO, Wandell BA. Population receptive field estimates in human visual cortex. Neurolmage. 2008;39(2):647-60.

28. Qi S, Mu YF, Cui LB, Li R, Shi M, Liu Y, et al. Association of Optic Radiation Integrity with Cortical Thickness in Children with Anisometropic Amblyopia. Neurosci Bull. 2016;32(1):51-60.

29. Li Q, Song M, Xu J, Qin W, Yu C, Jiang T. Cortical thickness development of human primary visual cortex related to the age of blindness onset. Brain Imaging Behav. 2016.

30. Park HJ, Lee JD, Kim EY, Park B, Oh MK, Lee S, et al. Morphological alterations 
in the congenital blind based on the analysis of cortical thickness and surface area. Neurolmage. 2009;47(1):98-106.

31. Duan Y, Norcia AM, Yeatman JD, Mezer A. The Structural Properties of Major White Matter Tracts in Strabismic Amblyopia. Investigative ophthalmology \& visual science. 2015;56(9):5152-60.

32. Allen B, Spiegel DP, Thompson B, Pestilli F, Rokers B. Altered white matter in early visual pathways of humans with amblyopia. Vision research. 2015;114:48-55.

33. Tailor V, Bossi M, Greenwood JA, Dahlmann-Noor A. Childhood amblyopia: current management and new trends. Br Med Bull. 2016.

34. Pediatric Eye Disease Investigator G. A randomized trial of atropine vs. patching for treatment of moderate amblyopia in children. Archives of ophthalmology. 2002;120(3):268-78.

35. Repka MX, Beck RW, Holmes JM, Birch EE, Chandler DL, Cotter SA, et al. A randomized trial of patching regimens for treatment of moderate amblyopia in children. Archives of ophthalmology. 2003;121(5):603-11.

36. Holmes JM, Kraker RT, Beck RW, Birch EE, Cotter SA, Everett DF, et al. A randomized trial of prescribed patching regimens for treatment of severe amblyopia in children. Ophthalmology. 2003;110(11):2075-87.

37. Scheiman MM, Hertle RW, Kraker RT, Beck RW, Birch EE, Felius J, et al. Patching vs atropine to treat amblyopia in children aged 7 to 12 years: a randomized trial. Archives of ophthalmology. 2008;126(12):1634-42.

38. Repka MX, Kraker RT, Beck RW, Birch E, Cotter SA, Holmes JM, et al. Treatment of severe amblyopia with weekend atropine: results from 2 randomized clinical trials. J Aapos. 2009;13(3):258-63.

39. Hess RF, Mansouri B, Thompson B. A new binocular approach to the treatment of amblyopia in adults well beyond the critical period of visual development. Restorative neurology and neuroscience. 2010;28(6):793-802.

40. Hess RF, Thompson B, Black JM, Machara G, Zhang P, Bobier WR, et al. An iPod treatment of amblyopia: an updated binocular approach. Optometry (St Louis, Mo. 2012;83(2):87-94.

41. Li SL, Jost RM, Morale SE, Stager DR, Dao L, Stager D, et al. A binocular iPad treatment for amblyopic children. Eye (London, England). 2014;28(10):1246-53.

42. Li SL, Jost RM, Morale SE, De La Cruz A, Dao L, Stager D, Jr., et al. Binocular iPad treatment of amblyopia for lasting improvement of visual acuity. JAMA ophthalmology. 2015;133(4):479-80.

43. Hess RF, Thompson B. Amblyopia and the binocular approach to its therapy. Vision research. 2015;114:4-16.

44. Tsirlin I, Colpa L, Goltz HC, Wong AM. Behavioral Training as New Treatment for Adult Amblyopia: A Meta-Analysis and Systematic Review. Investigative ophthalmology \& visual science. 2015;56(6):4061-75.

45. Tailor V, Bossi M, Bunce C, Greenwood JA, Dahlmann-Noor A. Binocular versus standard occlusion or blurring treatment for unilateral amblyopia in children aged three to eight years. Cochrane database of systematic reviews (Online). 2015;8:CD011347.

46. Spiegel DP, Li J, Hess RF, Byblow WD, Deng D, Yu M, et al. Transcranial direct current stimulation enhances recovery of stereopsis in adults with amblyopia. Neurotherapeutics : the journal of the American Society for Experimental NeuroTherapeutics. 2013;10(4):831-9.

47. Bossi M, Anderson E, Tailor V, Bex P, Greenwood J, Dahlmann-Noor A, et al., editors. An Exploratory Study of a Novel Home-Based Binocular Therapy for Childhood Amblyopia. ARVO; 2014.

48. Vedamurthy I, Nahum M, Bavelier D, Levi DM. Mechanisms of recovery of visual function in adult amblyopia through a tailored action video game. Scientific reports. 2015;5:8482.

49. Chen Z, Li J, Thompson B, Deng D, Yuan J, Chan L, et al. The effect of 
Bangerter filters on binocular function in observers with amblyopia. Investigative ophthalmology \& visual science. 2015;56(1):139-49.

50. Zhou J, Jia W, Huang CB, Hess RF. The effect of unilateral mean luminance on binocular combination in normal and amblyopic vision. Scientific reports.

2013;3:2012.

51. Ding J, Levi DM. Rebalancing binocular vision in amblyopia. Ophthalmic Physiol Opt. 2014;34(2):199-213.

52. Waddingham PE, Butler TK, Cobb SV, Moody AD, Comaish IF, Haworth SM, et al. Preliminary results from the use of the novel Interactive binocular treatment (I-BiT) system, in the treatment of strabismic and anisometropic amblyopia. Eye (London, England). 2006;20(3):375-8.

53. Herbison N, MacKeith D, Vivian A, Purdy J, Fakis A, Ash IM, et al. Randomised controlled trial of video clips and interactive games to improve vision in children with amblyopia using the I-BiT system. The British journal of ophthalmology. 2016.

54. $\quad$ Li RW, Tran TT, Craven AP, Leung TW, Chat SW, Levi DM. Sharpening coarseto-fine stereo vision by perceptual learning: asymmetric transfer across the spatial frequency spectrum. R Soc Open Sci. 2016;3(1):150523.

55. Levi DM, Li RW. Perceptual learning as a potential treatment for amblyopia: a mini-review. Vision research. 2009;49(21):2535-49.

56. Levi DM, Polat U. Neural plasticity in adults with amblyopia. Proceedings of the National Academy of Sciences of the United States of America. 1996;93(13):6830-4. 57. Polat U, Ma-Naim T, Belkin M, Sagi D. Improving vision in adult amblyopia by perceptual learning. Proceedings of the National Academy of Sciences of the United States of America. 2004;101(17):6692-7.

58. Chen Z, Li J, Liu J, Cai X, Yuan J, Deng D, et al. Monocular perceptual learning of contrast detection facilitates binocular combination in adults with anisometropic amblyopia. Scientific reports. 2016;6:20187.

59. Ren Z, Zhou J, Yao Z, Wang Z, Yuan N, Xu G, et al. Neuronal basis of perceptual learning in striate cortex. Scientific reports. 2016;6:24769.

60. Li RW, Ngo C, Nguyen J, Levi DM. Video-game play induces plasticity in the visual system of adults with amblyopia. PLoS biology. 2011;9(8):e1001135.

61. Bejjanki VR, Zhang R, Li R, Pouget A, Green CS, Lu ZL, et al. Action video game play facilitates the development of better perceptual templates. Proceedings of the National Academy of Sciences of the United States of America.

2014;111(47):16961-6.

62. Zhou J, Clavagnier S, Hess RF. Short-term monocular deprivation strengthens the patched eye's contribution to binocular combination. Journal of vision. 2013;13(5). 63. Duffy KR, Mitchell DE. Darkness alters maturation of visual cortex and promotes fast recovery from monocular deprivation. Curr Biol. 2013;23(5):382-6.

64. Thompson B, Mansouri B, Koski L, Hess RF. Brain plasticity in the adult: modulation of function in amblyopia with rTMS. Curr Biol. 2008;18(14):1067-71.

65. Clavagnier S, Thompson B, Hess RF. Long lasting effects of daily theta burst rTMS sessions in the human amblyopic cortex. Brain Stimul. 2013;6(6):860-7.

66. Spiegel DP, Byblow WD, Hess RF, Thompson B. Anodal transcranial direct current stimulation transiently improves contrast sensitivity and normalizes visual cortex activation in individuals with amblyopia. Neurorehabilitation and neural repair. 2013;27(8):760-9.

67. Pawar PV, Mumbare SS, Patil MS, Ramakrishnan S. Effectiveness of the addition of citicoline to patching in the treatment of amblyopia around visual maturity: a randomized controlled trial. Indian J Ophthalmol. 2014;62(2):124-9.

68. Pediatric Eye Disease Investigator G, Repka MX, Kraker RT, Dean TW, Beck RW, Siatkowski RM, et al. A randomized trial of levodopa as treatment for residual amblyopia in older children. Ophthalmology. 2015;122(5):874-81.

69. Maya Vetencourt JF, Sale A, Viegi A, Baroncelli L, De Pasquale R, O'Leary OF, et al. The antidepressant fluoxetine restores plasticity in the adult visual cortex. 
Science (New York, NY. 2008;320(5874):385-8.

70. Beshara S, Beston BR, Pinto JG, Murphy KM. Effects of Fluoxetine and Visual Experience on Glutamatergic and GABAergic Synaptic Proteins in Adult Rat Visual Cortex. eNeuro. 2015;2(6).

71. Southwell DG, Froemke RC, Alvarez-Buylla A, Stryker MP, Gandhi SP. Cortical plasticity induced by inhibitory neuron transplantation. Science (New York, NY. 2010;327(5969):1145-8.

72. Davis MF, Figueroa Velez DX, Guevarra RP, Yang MC, Habeeb M, Carathedathu MC, et al. Inhibitory Neuron Transplantation into Adult Visual Cortex Creates a New Critical Period that Rescues Impaired Vision. Neuron. 2015;86(4):1055-66.

73. Bochner DN, Sapp RW, Adelson JD, Zhang S, Lee H, Djurisic M, et al. Blocking PirB up-regulates spines and functional synapses to unlock visual cortical plasticity and facilitate recovery from amblyopia. Sci TransI Med. 2014;6(258):258ra140.

74. Pizzorusso T, Medini P, Berardi N, Chierzi S, Fawcett JW, Maffei L. Reactivation of ocular dominance plasticity in the adult visual cortex. Science (New York, NY. 2002;298(5596):1248-51.

75. Vorobyov V, Kwok JC, Fawcett JW, Sengpiel F. Effects of digesting chondroitin sulfate proteoglycans on plasticity in cat primary visual cortex. J Neurosci.

2013;33(1):234-43.

76. Gruzensky WD, Palmer EA. Intractable diplopia: a clinical perspective. Graefe's archive for clinical and experimental ophthalmology = Albrecht von Graefes Archiv fur klinische und experimentelle Ophthalmologie. 1988;226(2):187-92.

77. Bielschowsky A. Congenital and acquired disruption of fusion. American journal of ophthalmology. 1935;18:925-37.

78. Piano M, Newsham D. A pilot study examining density of suppression measurement in strabismus. Strabismus. 2015;23(1):14-21.

79. Newsham D, O'Connor AR. Assessment of the Density of Suppression to Identify Risk of Intractable Diplopia in the United Kingdom. Strabismus. 2016;24(2):4550.

80. Kehrein S, Kohnen T, Fronius M. Dynamics of Interocular Suppression in Amblyopic Children during Electronically Monitored Occlusion Therapy: First Insight. Strabismus. 2016;24(2):51-62. 